\title{
Linear Filtering
}

\author{
By Ralph Shapiro
}

\begin{abstract}
A simple linear smoothing operator of general order $p$ is constructed for discretized functions. It is particularly suited for problems in numerical integration where it is necessary or desirable to suppress two-grid-interval waves. The operator is ideal in the sense that for the class of damping operators of order $p$, which remove two-gridinterval waves without changing the phase of any wave component, this operator produces the least damping of the amplitudes of all other waves.
\end{abstract}

1. Introduction. The avoidance of nonlinear computational instability in numerical integrations of finite-difference approximations of systems of nonlinear partial differential equations, such as those arising in fluid dynamics, often requires the use of smoothing. The smoothing may be implicit in the finite-difference procedure or explicit as a modification of the differential equation. In either case, repeated application of the smoothing operator may produce serious damping of physically significant wave components. To minimize such degradation of the solution, it is desirable to limit the smoothing as far as possible to those wave components most affected by instability, that is, the high frequency or high wave number components. One way of accomplishing this result is to use a finite-difference operator which avoids implicit smoothing as far as possible and to incorporate a highly scale-dependent explicit smoothing operator. The linear filtering operator discussed below was designed for this purpose.

2. Smoothing Operator. Let $f(x)$ represent some function of time or space in one dimension, integrable in the finite domain $-d<x<d$, and let $f\left(x_{i}\right)=f_{i}$ for discrete values of $x$ such that $x_{i}=i \Delta x$ where $i$ is an integer and $\Delta x>0$ is such that $2 d / \Delta x$ is an integer. We define the simple three-point symmetrical operation

$$
f_{i}^{(1)}=1 / 4\left(f_{i-1}+2 f_{i}+f_{i+1}\right)=\left(1+\delta^{2} / 4\right) f_{i},
$$

where we have scaled the interval $(-d, d)$ so that $\Delta x=1$, and where $\delta f_{i}=\left(f_{i+1 / 2}-f_{i-1 / 2}\right)$. $f\left(x_{i}\right)$ can be expressed in terms of a sum of Fourier components of the general form $A_{n} \cos n\left(x_{i}-\varphi_{n}\right)$ where $A_{n}$ is the amplitude of the wave component with wave number $n$ ( $n=2 \pi / \lambda$, where $\lambda$ is the wavelength of the component), and $\varphi_{n}$ is the phase of the component. If $A_{n}$ is the $n$th component of $f_{i}$, and $A_{n}^{(1)}$ is the $n$th component of $f_{i}^{(1)}$, then

$$
A_{n}^{(1)}=\left[1-\sin ^{2}(n \Delta x / 2)\right] A_{n} .
$$

Thus, the operator $\left(1+\delta^{2} / 4\right)$ defined in (1) damps the amplitude of each of the Fourier components of $f_{i}$ by the factor $\left[1-\sin ^{2}(n \Delta x / 2)\right]$. This factor is called the amplitude response function of the operator (1). 
It is apparent from (2) that the operator represented by (1) removes the shortest resolvable wave component (period or length $2 \Delta x$ ) and preserves the phase of the discretized function $f_{i}$. However, even relatively few applications of the operation (1) will produce considerable damping of components 5 to 10 times this length (see Table 1 for $p=0$ ). To maintain the desirable properties of (1) and yet avoid appreciable damping of longer wave components, Shapiro [3] suggested a higher order operator whose amplitude response is

$$
R_{k}(n)=1-\sin ^{2 k} \alpha
$$

where $k=2^{j}, j$ is any nonnegative integer, and where $\alpha=n \Delta x / 2$. For any order $k$, the operator represented by (3) is constructed by convolving (1) with a sequence of $(k-1)$ three-point symmetrical amplifying operators, comparable in form to (1).

If $f_{i}^{(k)}$ is the operator whose amplitude response is $R_{k}$, then

$$
\begin{aligned}
f_{i}^{(1)} & =\left(1+\delta^{2} / 4\right) f_{i}, \\
f_{i}^{(2)} & =\left(1-\delta^{2} / 4\right)\left(1+\delta^{2} / 4\right) f_{i}, \\
f_{i}^{(4)} & =\left(1+\delta^{4} / 16\right)\left(1-\delta^{4} / 16\right) f_{i}, \\
\dot{ } & \dot{\cdot} \\
f_{i}^{(k)} & =\left[1+(\delta / 2)^{k}\right]\left[1-(\delta / 2)^{k}\right] f_{i} .
\end{aligned}
$$

It was shown in [3] that such operators, for any order $k$, remove $2 \Delta x$ waves completely but minimize the damping of all longer wave components. Such high order operators have been adapted for use in numerical models of the large-scale atmospheric circulation where they approximate the characteristics of a highly scale-dependent nonlinear diffusion in the differential equation (Shapiro [4], Hunt [1] and [2]). The disadvantage of these operators is that they are defined only for integer values of $j$. Since the number of points in the combined operator is $(2 k+1)$, this means that only operators of 3,5 , $9,17, \ldots,\left(1+2^{j+1}\right)$ points are defined. There are many applications in which operators of an intermediate size would be useful, as, for example, when applying the operator at gridpoints approaching a boundary. This disadvantage can easily be overcome by a modification suggested by E. Knighting, G. Corby and A. Gilchrist (personal communication). Their approach is to construct the higher order operator by using a truncation of the infinite series formally representing the inverse of $\left(1+\delta^{2} / 4\right)$.

The inverse of $\left(1+\delta^{2} / 4\right)$ is given by the infinite series

$$
\left(1+\delta^{2} / 4\right)^{-1}=1-(\delta / 2)^{2}+(\delta / 2)^{4}-(\delta / 2)^{6}+\cdots \cdot
$$

It is obvious that if we operate on $f_{i}^{(1)}$ with any finite truncation of the series (5) we can restore the amplitude of any wave component (not specifically eliminated by the operation (1)) as closely as we wish. Thus,

$$
f_{i}^{(p+1)}=\left[1+(\delta / 2)^{2}\right]\left[1-(\delta / 2)^{2}+(\delta / 2)^{4}+\cdots+(-1)^{p}(\delta / 2)^{2 p}\right] f_{i}
$$

indicates a restoration of $f_{i}^{(1)}$ to the order $p$ and yields an operator with $2 p+3$ terms. If we expand (6), we find 


$$
f_{i}^{(p+1)}=\left[1+(-1)^{p}(\delta / 2)^{2 p+2}\right] f_{i},
$$

where $p=0,1,2,3, \ldots$.

Since the stencil for $\delta^{2 q} f_{i}$ for any integer $q$ is given by the binomial coefficients, the stencil for $f_{i}^{(p+1)}$ can be obtained directly. That is,

$$
\delta^{2 q} f_{i}=(-1)^{q}\left(\begin{array}{c}
2 q \\
q
\end{array}\right) f_{i}+\sum_{j=0}^{q-1}(-1)^{j}\left(\begin{array}{c}
2 q \\
j
\end{array}\right)\left[f_{i+(q-j)}+f_{i-(q-j)}\right],
$$

where $\left(\begin{array}{c}n \\ m\end{array}\right)=n ! /(n-m) ! m !$. Thus,

$$
\begin{aligned}
f_{i}^{(p+1)}= & {\left[1-\left(1 / 2^{2 p+2}\right)\left(\begin{array}{c}
2 p+2 \\
p+1
\end{array}\right)\right] f_{i} } \\
& +\left[(-1)^{p} / 2^{2 p+2}\right] \sum_{j=0}^{p}(-1)^{j}\left(\begin{array}{c}
2 p+2 \\
j
\end{array}\right)\left[f_{i+(1+p-j)}+f_{i-(1+p-j)}\right] .
\end{aligned}
$$

$R_{p+1}$, the amplitude response function for the operator (9) is obtained from (6) by making use of the fact that the. Fourier representation of the operator $\delta^{2}$ is $\left(-4 \sin ^{2} \alpha\right)$. Therefore,

$$
R_{p+1}=\left|f_{i}^{(p+1)}\right| f_{i} \mid=\left(1-\sin ^{2} \alpha\right)\left(1+\sin ^{2} \alpha+\sin ^{4} \alpha+\cdots+\sin ^{2 p} \alpha\right) .
$$

Consequently,

$$
R_{p+1}=1-\sin ^{2 p+2} \alpha
$$

For those values of $k=p+1=2^{j}$, Eqs. (11) and (3) and their corresponding operators are identical. It should be noted that Eqs. (10) and (11) could have been obtained directly from (2) by operating on $\left(1-\sin ^{2} \alpha\right)$ with its inverse.

It is obvious from (11) that $R_{p+1}$ is restricted such that $1 \geqslant R_{p+1} \geqslant R_{p} \geqslant 0$. Furthermore, we shall prove that $R_{p+1}$ is the amplitude response function which for any $p$ yields the maximum restoration of amplitude of each wave component damped by $f_{i}^{(1)}$ without amplifying any component beyond its original value and without changing the phase of any component.

TABle 1. Values of the Amplitude Response, $R_{p+1}=1-\sin ^{2 p+2} \alpha$ for Various Orders $p$, for Various Wavelengths $\lambda$

\begin{tabular}{ccccccc}
\hline \hline & \multicolumn{5}{c}{$p$} \\
$\lambda / \Delta x$ & 0 & 1 & 2 & 3 & 4 & 5 \\
\hline 2 & 0 & 0 & 0 & 0 & 0 & 0 \\
3 & 0.25000 & 0.43750 & 0.57812 & 0.68359 & 0.76270 & 0.82202 \\
4 & 0.50000 & 0.75000 & 0.87500 & 0.93750 & 0.96875 & 0.98438 \\
6 & 0.75000 & 0.93750 & 0.98438 & 0.99609 & 0.99902 & 0.99976 \\
10 & 0.90451 & 0.99088 & 0.99913 & 0.99992 & 0.99999 & 1.00000 \\
15 & 0.95677 & 0.99813 & 0.99992 & 1.00000 & 1.00000 & 1.00000 \\
20 & 0.97553 & 0.99940 & 0.99999 & 1.00000 & 1.00000 & 1.00000 \\
\hline
\end{tabular}


THEOREM. Given $1 \geqslant R_{p+1}^{*} \geqslant 0$ where $R_{p+1}^{*}$ has the form

$R_{p+1}^{*}=\left(1-\sin ^{2} \alpha\right) P_{p}^{*}=\left(1-\sin ^{2} \alpha\right)\left(1+A_{1} \sin ^{2} \alpha+A_{2} \sin ^{4} \alpha+\cdots+A_{p} \sin ^{2 p} \alpha\right)$

where $A_{j} \geqslant 0$, then $R_{p+1} \geqslant R_{p+1}^{*}$ for any $p$.

Proof. We are given $1=\left(1-\sin ^{2} \alpha\right)\left(1-\sin ^{2} \alpha\right)^{-1} \geqslant R_{p+1}^{*}=\left(1-\sin ^{2} \alpha\right) P_{p}^{*}$ $\geqslant 0$ : Furthermore, from (11) we have $\left(1-\sin ^{2} \alpha\right)^{-1}=P_{p} /\left(1-\sin ^{2 p+2} \alpha\right)$, where $P_{p}=1+\sin ^{2} \alpha+\sin ^{4} \alpha+\cdots+\sin ^{2 p} \alpha$. Therefore, we have $1 \geqslant\left(P_{p}^{*} / P_{p}\right)\left(1-\sin ^{2 p+2} \alpha\right) \geqslant 0$. If for any $p$ the ratio $P_{p}^{*} / P_{p} \leqslant 1$, then $R_{p+1} \geqslant R_{p+1}^{*}$. But $P_{p}^{*} / P_{p}>1$ is not permitted for any $p$ since if it were, then for some sufficiently small $\sin ^{2} \alpha$, the product $\left(P_{p}^{*} / P_{p}\right)\left(1-\sin ^{2 p+2} \alpha\right)$ would exceed unity.

Some notion of the efficiency of $f_{i}^{(p+1)}$ can be obtained from Table 1 which lists the values of $R_{p+1}$ for waves of various lengths for increasing values of $p$. The stencils for $f_{i}^{(p+1)}$ for values of $p$ up to 9 are given in Table 2. Smoothing in more than one dimension can easily be performed by applying the one-dimensional operator (9) separately in each dimension. Although the operator $f_{i}^{(p+1)}$ was designed for use where repeated smoothing applications are required, it may also find more general use as an efficient and highly scale-dependent filter.

TABLE 2. Stencils for $f_{i}^{(p+1)}$ for Various Values of $p$

\begin{tabular}{|c|c|c|c|c|c|c|c|c|c|c|c|c|}
\hline$p$ & & $f_{i}$ & $f_{i \pm 1}$ & $f_{i \pm 2}$ & $f_{i \pm 3}$ & $f_{i \pm 4}$ & $f_{i \pm 5}$ & $f_{i \pm 6}$ & $f_{i \pm 7}$ & $f_{i \pm 8}$ & $f_{i \pm 9}$ & $f_{i \pm 10}$ \\
\hline 0 & $1 / 2^{2}$ & (2 & 1) & & & & & & & & & \\
\hline 1 & $1 / 2^{4}$ & $(10$ & 4 & $-1)$ & & & & & & & & \\
\hline 2 & $1 / 2^{6}$ & $(44$ & 15 & -6 & 1) & & & & & & & \\
\hline 3 & $1 / 2^{8}$ & (186 & 56 & -28 & 8 & $-1)$ & & & & & & \\
\hline 4 & $1 / 2^{10}$ & (772 & 210 & -120 & 45 & -10 & 1) & & & & & \\
\hline 5 & $1 / 2^{12}$ & $(3172$ & 792 & -495 & 220 & -66 & 12 & $-1)$ & & & & \\
\hline 6 & $1 / 2^{14}$ & (12952 & 3003 & -2002 & 1001 & -364 & 91 & -14 & 1) & & & \\
\hline 7 & $1 / 2^{16}$ & (52666 & 11440 & -8008 & 4368 & -1820 & 560 & -120 & 16 & $-1)$ & & \\
\hline 8 & $1 / 2^{18}$ & (213524 & 43758 & -31824 & 18564 & -8568 & 3060 & -816 & 153 & -18 & 1) & \\
\hline 9 & $1 / 2^{20}$ & (863820 & 167960 & -125970 & 77520 & -38760 & 15504 & -4845 & 1140 & -190 & 20 & $-1)$ \\
\hline
\end{tabular}

Meteorology Laboratory

Air Force Cambridge Research Laboratories

Bedford, Massachusetts 01731

1. B. G. HUNT, "Zonally symmetric global general circulation models with and without the hydrologic cycle," Tellus, v. 25, 1973, pp. 337-354.

2. B. G. HUNT, "A global general circulation model of the atmosphere based on the semispectral method," Monthly Weather Review, v. 102, 1974, pp. 3-16.

3. R. SHAPIRO, "Smoothing, filtering, and boundary effects," Rev. Geophys. and Space Phys., v. 8, 1970, pp. 359-387.

4. R. SHAPIRO, "The use of linear filtering as a parameterization of atmospheric diffusion," J. Atmospheric Sci., v. 28, 1971, pp. 523-531. 